\title{
BMJ Open Descriptive analysis of real-world medication use pattern of statins and antiplatelet agents among patients with acute coronary syndrome in Hong Kong and the USA
}

\author{
Yun Wang, ${ }^{\oplus 1}$ Michael B Nichol, ${ }^{2}$ Bryan PY Yan, ${ }^{3,4}$ Joanne Wu, ${ }^{2}$ Brian Tomlinson, ${ }^{3,4}$ \\ Vivian WY Lee ${ }^{\circ}$
}

To cite: Wang Y, Nichol MB, Yan BPY, et al. Descriptive analysis of real-world medication use pattern of statins and antiplatelet agents among patients with acute coronary syndrome in Hong Kong and the USA. BMJ Open 2019;9:e024937. doi:10.1136/ bmjopen-2018-024937

- Prepublication history for this paper is available online. To view these files, please visit the journal online (http://dx.doi. org/10.1136/bmjopen-2018024937).

Received 8 July 2018

Revised 31 May 2019

Accepted 10 June 2019

A) Check for updates

(C) Author(s) (or their employer(s)) 2019. Re-use permitted under CC BY-NC. No commercial re-use. See rights and permissions. Published by BMJ.

For numbered affiliations see end of article.

Correspondence to

Dr Vivian WY Lee;

vivianlee@cuhk.edu.hk

\section{ABSTRACT}

Objectives The objective was to explore the differences in medication use pattern of lipid-lowering drug (LLD) and antiplatelet agents among post-percutaneous coronary intervention patients with acute coronary syndrome aged $<65$ in Hong Kong (HK) and the USA.

Design Retrospective study.

Setting This study used deidentified claims data from Clinformatics Data Mart database (Optumlnsight, Eden Prairie, Minnesota, USA) and electronic health records from HK Hospital Authority Clinical Data Analysis and Reporting System database.

Participants We used 1 year prescription records of LLDs and antiplatelet agents among 1013 USA patients and 270 HK Chinese patients in 2011-2013.

Primary and secondary outcome measures Continuity was investigated on the assumption that one defined daily dose represented 1 day treatment. Medication possession ratio method was used to evaluate the adherence. Multivariate-adjusted logistic regressions were constructed to compare the good continuity and adherence levels in the merged database with the cutoffs set at $80 \%$, and Cox proportional hazard models were built using the time to discontinuation as the dependent variable, to assess the persistence level.

Results HK Chinese patients were less adherent $(67.41 \%$ vs $84.60 \%$, adjusted odds ratio (AOR) for Americans over Chinese $=2.23(95 \% \mathrm{Cl}=1.60$ to 3.12$), \mathrm{p}<0.001)$ to antiplatelet agents compared with American patients but better adherent to statins $(90.00 \%$ vs $78.18 \%, A 0 R=0.37$ ( 0.23 to 0.58$), p<0.001)$. The discontinuation with statins was more common in American patients (13.33\% vs $34.25 \%$, adjusted hazard ratio $(\mathrm{AHR})=2.95(2.05$ to 4.24), $\mathrm{p}<0.001)$. Low-to-moderate potency statins and clopidogrel were favoured by our HK local physicians, while American patients received higher doses of statins and prasugrel.

Conclusions We seemed to find HK physicians tended to prescribe cheaper and lower doses of statins and antiplatelet agents when compared with the privately insured patients in the USA, though the adherence and persistence levels of $\mathrm{HK}$ patients with statins were relatively good.

\section{Strengths and limitations of this study}

- The cost-driven prescription behaviour was a possibility in Hong Kong, but little was known about the prescribing behaviour of statins and antiplatelet agents in the management of young cardiovascular disease patients.

- Our research shed some light on the real-world drug utilisation of lipid-lowering drug (LLD) and antiplatelet agents (clopidogrel, ticagrelor and prasugrel) among acute coronary syndrome patients $<65$ years in HK public hospitals, compared with a privately and commercially insured US patient cohort.

- We identified the adherence, continuity and discontinuation patterns of HK patients, compared with a typical American privately insured cohort.

- We seemed to find HK physicians tended to prescribe cheaper and lower doses of LLDs and antiplatelet agents compared with the privately insured patients in the USA.

\section{INTRODUCTION}

Hong Kong (HK) was thought to be one of the most efficient healthcare systems in the world, ${ }^{1}$ with a life expectancy of 84 years in $2016^{2}$ and publicly subsidised public hospitals virtually free to all citizens. It had a population of 7.35 million, accompanying $5.7 \%$ of gross domestic product on health expenditure in $2017 .^{2}$ In contrast, the USA spent nearly 4.52 times as much per capita on healthcare as HK. ${ }^{13}$ The government-funded universal healthcare is the cornerstone of HK healthcare system. Universal healthcare provides equal access to medical services to all citizens, and the government controls the price of medication and medical services through negotiation and regulation. In HK, the inpatient hospital stay costed Hong Kong Dollars (HKD) 120 (1 US dollar (USD)=7.8 HKD) 
per day for a patient's out-of-pocket spending in $2019 .{ }^{4}$ Patients paid 135 HKDs for their first specialist outpatient consultation and 80 HKD per time for the subsequent attendances with an additional 15 HKD per drug item. ${ }^{4}$ The drawback with the universal healthcare system is the government focuses on providing basic healthcare. ${ }^{156}$ There is a high possibility that healthcare budget had a ceiling and the government funding decisions are mainly based on cost-saving strategy. ${ }^{7}$ In America, it was widely believed that the privately insured patients covered by a commercial health plan were more likely to receive better medical care than the publicly insured ${ }^{89}$ In comparison, the government may limit costly services to cut costs for publicly insured patients, which made the underprescribing/treatment a possibility, as reported before ${ }^{10-12}$ in HK.

HK healthcare system by large followed UK National Health Service (NHS), for it was a British colony for 155 years. Where it lacked from the UK system was mostly clinical audit and quality assurance. UK claimed its commitment in the 2014 Pharmaceutical Price Regulation Scheme, ${ }^{13}$ that 'the UK should compare itself with other countries if it is to deliver a world-class NHS' and 'develop and evolve an approach to the analysis and publication of comparative information on international medicines use on a periodic basis'. When this was compared with HK, where public health services were facing the same challenges, a small effort was made to make an international comparison to identify the underlying issues regarding the medication use pattern.

In recent years, risk factors associated with lifestyles has gradually led to the prevalence of cardiovascular diseases in young adults aged $<65$ worldwide. ${ }^{14}$ However, young patients were rarely studied, ${ }^{15}$ and little was known about how the young patients were managed after they were identified as atherosclerotic cardiovascular disease (ASCVD) patients. There was no study comparing the prescribing patterns of cardiovascular drugs in HK government-subsidised system with those in the private health insurance system presumably due to the difficulty in obtaining useful data and that HK does not have a private healthcare system which is transparent and well developed compared with other countries on this agenda. In terms of the delivery of care, $90 \%$ of healthcare services in HK were provided by 44 public hospitals. ${ }^{16}$ In HK, public and private healthcare operate in separate systems, and the main legislation on data protection limited the sharing system between the public and private sectors, which made it less feasible to extract patients' information from any private insurance providers for the comparison purpose.

Lipid-lowering drugs (LLDs) and antiplatelet agents were found to be the least adherent medications in post-percutaneous coronary intervention (PCI) acute coronary syndrome (ACS) patients from our pilot study. ${ }^{17}$ Therefore, in this study, we chose these two worst-adhered classes of cardiovascular drugs as the focus. There is a rich literature to describe the local prescribing patterns of $\operatorname{statin}^{18}$ and antiplatelet ${ }^{19}$ agents in HK. However, the international comparison between $\mathrm{HK}$ and other countries is lacking. Clinician in HK mainly followed the US and European guidelines as many other countries did, ${ }^{20}$ but they were meanwhile aware of the emerging clinical evidence and recommendations by mainland China. That may sometimes put them in a dilemma ${ }^{21}$ at the absence of nuanced guidance on specific scenarios. For example, the mainland Chinese guideline held a conservative attitude against high-potency statin, ${ }^{22}$ while the most recent American guideline highly recommended the use of high-potency statins. ${ }^{23}$

In the current research, with a limited health budget available for pharmacy in HK, we attempted to identify the medication use patterns of HK patients, compared with privately insured Americans, a typical cohort which should be prescribed sufficient drugs ${ }^{24}$ accompanying the rapid updating of guidelines by American College of Cardiology/American Heart Association. A broad spectrum of factors is likely to lead to observed differences between HK Chinese and American patients. This type of comparison mainly enabled the health policy makers and health practitioners in HK to compare itself with other countries, and to understand the potentially mixed effects from both healthcare systems and the contextual difference ${ }^{25}$ in implementing the clinical practice guidelines for Chinese patients.

\section{METHODS}

This was a retrospective and comparative analysis using two cohorts: one from HK Hospital Authority (HA) Clinical Data Analysis and Reporting System (CDARS) database, representing all the local Chinese citizens in the New Territories East Cluster admitted to a PCI-capable acute public hospital; and the other from the $1 \%$ random cohort of the deidentified Clinformatics Data Mart database (OptumInsight, Eden Prairie, Minnesota, USA), which was referred as 'Optum' below, comprising administrative claims data for commercially insured multiethnic population in the parallel inclusion period between January 2011 and December 2012. New Territories East Cluster is one of the seven hospital clusters managed by Hospital Authority in HK, which accounted for roughly $7 \%$ of Hong Kong's population and took up $10 \%$ of the health budget of the government per year. ${ }^{26}$

Our inclusion criterion was, the study cohorts in both databases should be ACS patients identified by International Classification of Diseases, Ninth Revision, Clinical Modification (ICD-9-CM) diagnosis codes of 411. $\mathrm{xx}$ (unstable angina) and 410.xx (myocardial infarction), ${ }^{27} 28 \geq 21$ years who received a first documented PCI (ICD-9-CM procedure codes $36.0 \mathrm{x}^{29}$ ) during the inclusion period between January 2011 and December 2012, and were continuously enrolled in the respective database for 1 year after their index PCI. The patients who had any prior ACS diagnosis or underwent PCI in the preceding 6 months were excluded. The available LLDs in HK localised HA Drug Formulary were atorvastatin, 
Table 1 DDD defined by WHO

\begin{tabular}{|c|c|c|c|c|}
\hline ATC code & Name & DDD & Unit & $\begin{array}{l}\text { Administrative } \\
\text { route }\end{array}$ \\
\hline B01AC06 & $\begin{array}{l}\text { Acetylsalicylic } \\
\text { acid }\end{array}$ & 1 & tablet & Oral \\
\hline B01AC04 & Clopidogrel & 75 & mg & Oral \\
\hline B01AC22 & Prasugrel & 10 & $\mathrm{mg}$ & Oral \\
\hline B01AC24 & Ticagrelor & 0.18 & g & Oral \\
\hline C10AA05 & Atorvastatin & 20 & $\mathrm{mg}$ & Oral \\
\hline C10AA04 & Fluvastatin & 60 & $\mathrm{mg}$ & Oral \\
\hline C10AA02 & Lovastatin & 45 & $\mathrm{mg}$ & Oral \\
\hline C10AA03 & Pravastatin & 30 & $\mathrm{mg}$ & Oral \\
\hline C10AA07 & Rosuvastatin & 10 & $\mathrm{mg}$ & Oral \\
\hline C10AA01 & Simvastatin & 30 & $\mathrm{mg}$ & Oral \\
\hline$C 10 A \times 09$ & Ezetimibe & 10 & $\mathrm{mg}$ & Oral \\
\hline C10AC01 & Colestyramine & 14 & $g$ & Oral \\
\hline C10AD02 & Nicotinic acid & 2 & g & Oral \\
\hline C10AB05 & Fenofibrate & 0.2 & g & Oral \\
\hline C10AB04 & Gemfibrozil & 1.2 & g & Oral \\
\hline
\end{tabular}

ATC, anatomical therapeutic chemical; DDD, daily defined dose.

cholestyramine, ezetimibe, fenofibrate, fluvastatin, gemfibrozil, rosuvastatin, simvastatin and evolocumab. The LLDs in Optum were identified through the American Hospital Formulary Service Therapeutic Class codes. The antiplatelet agents under investigation were clopidogrel, ticagrelor and prasugrel in both databases. Since aspirin was an over-the-counter medication in the USA and the non-prescription drugs taken by the patients were not captured in claims data, we chose not to include aspirin in this analysis.

The patients' age, sex, procedures, prescriptions, and visits to outpatient and inpatient clinics were extracted from both systems during the study period between -6 months and +12 months of the index procedure. The analysis of all prescriptions was made within 12 months that followed from the index PCI, and both entities had the advantage of making a follow-up of patients' prescriptions.

The utilisation of all drugs was expressed as the number of defined daily doses (DDDs)/inhabitant/year, a standard unit (table 1) calculated according to the Anatomical Therapeutic Chemical (ATC)/DDD methodology, ${ }^{30}$ which allowed the international comparison between countries and regions and quantified the annual prevalence of use. We evaluated the continuity under the assumption that 1 DDD represented 1 day treatment, and the patients should get $365 \mathrm{DDDs}^{31}$ within 1year in the respective databases. The limit between good and poor continuity was defined as $80 \% .^{31}$ The measurement of adherence was reported as medication possession ratio (MPR) ${ }^{32}$ the sum of the days' supply for all fills of a given drug in a particular time period divided by the number of days in the study window, and was evaluated to report the percentage of the time when a patient had medication available. Patients were deemed non-adherent if the MPR value was $<80 \%$. ${ }^{32}$ We also assessed the persistence level ${ }^{33}$ by the time to discontinuation as other did before, ${ }^{34}$ namely the time from first having the prescription dispensed to the discontinuation day (defined as with a $>14$ days' gap from the recent refill record).

\section{Statistical analysis}

Because the underlying factors (such as age, sex, comorbidities of diabetes (ICD code of 250.xx, 362.0x, 366.41) and hypertension (ICD code of 401.x-405.x, 362.11, 437.2) and prior cardiovascular disease history (ICD code of 390.x-459.x)) between Chinese and American patient cohorts could lead to variation in prescriptions, the multivariable logistic regressions adjusted for these covariates were used to test if the good continuity and adherence with statins and antiplatelet agents varied between these two cohorts in the merged database. Good continuity and adherence were the dichotomous outcomes in the logistic regressions, and the patient cohorts (USA vs HK) was analysed as a categorical variable.

Moreover, we conducted sensitivity analyses through estimating the average treatment effect (ATE) and the average treatment effect for the treated (ATT) on the basis of the abovementioned covariates age, sex, comorbidities of diabetes and hypertension, and prior cardiovascular disease history and assessing the continuity and adherence in the logistic regressions by propensity score (PS) weighting for ATT or ATE, ${ }^{35} 36$ because HK and American cohorts could differ substantially across the baseline characteristics. We did PS weighting, instead of PS matching, because normally PS matching would result in a significant reduction in the cohorts size. ${ }^{37}$ Multivariate-adjusted and PS-weighted Cox proportional hazards models were constructed for the outcome time to discontinuation, after adjustment for the covariates in the merged database. Patients were censored if they died or reached the end of the 1-year follow-up.

All statistical analyses were carried out using STATA V.14.0 (Stata Corp LP, College Station, Texas, USA).

\section{Patient and public involvement}

This research did not involve a prospective intervention to any human subjects. Both datasets were deidentified. The major findings through this international comparison could add knowledge to the existing literature and will be disseminated to the public in HK.

\section{RESULTS}

We identified 270 Chinese ACS patients from HK CDARS database and 1013 American patients from US Optum. Table 2 shows the baseline characteristics of the two cohorts. Apparently, American patients tend to carry a higher prevalence of hypertension than Chinese $(44.13 \%$ vs $12.22 \%$ in table 2 ). The rate of prior cardiovascular 
Table 2 Baseline characteristics of Hong Kong Chinese patients and American patients in Optum

\begin{tabular}{|c|c|c|c|}
\hline & Hong Kong CDARS - Chinese & US Optum - American & $P$ value \\
\hline Total & 270 & 1013 & \\
\hline Gender, no. (\%) & & & 0.001 \\
\hline Female & 33 (12.22) & $216(21.32)$ & \\
\hline Age, years, mean $\pm S D$ & $57.69(5.61)$ & $57.12(6.11)$ & 0.168 \\
\hline Diabetes & $36(13.33)$ & $114(11.25)$ & 0.345 \\
\hline Hypertension & $33(12.22)$ & $447(44.13)$ & $<0.001$ \\
\hline Prior cardiovascular disease & $33(12.22)$ & $32(3.16)$ & $<0.001$ \\
\hline
\end{tabular}

CDARS, Clinical Data Analysis and Reporting System; SD, Standard Deviation.

disease history in Chinese was much higher (12.22\%) than that of American patients (3.16\%). ACS patients in both cohorts were more likely to be men $(78.68 \%$ American and $87.78 \%$ Chinese) and have diabetes at the index day (13.33\% American and $11.25 \%$ Chinese).

Table 3 classifies the use of LLDs and antiplatelet agents in these two cohorts: the majority $(97.41 \%$ of Chinese and $95.26 \%$ of American) receive at least one prescription of statins. The average DDDs/inhabitant/ year of LLDs in American patients is almost twice of HK patients. Non-statin LLDs account for about $10 \%$ of the annual use of LLDs in the USA, but the use of non-statin drugs is rare $(0.47 \%)$ in HK (table 3 ). In HK, simvastatin is widely used $(65.78 \%$ of the annual DDDs of statins) as the initial dosing strategy $(85.93 \%)$, followed by relatively little use of rosuvastatin $(7.04 \%)$ and atorvastatin $(4.44 \%)$. In contrast, in Optum, atorvastatin was more prevalent (59.38\% of the annual DDDs of statins). Almost equivalent numbers of American patients were first put on atorvastatin (39.68\%) and simvastatin (35.64\%). Ever use of antiplatelet agents was found in $100 \%$ of HK patients and $96.94 \%$ of American patients, respectively. The annual use of antiplatelet agents was almost the same as HK Chinese patients (331.74 vs 326.62 DDDs/ inhabitant/year). Clopidogrel was highly preferred in Chinese patients, taking up $88.37 \%$ of the annual DDDs of antiplatelet agents. In the USA, only about the half of the annual DDDs belonged to clopidogrel and the other $32.65 \%$ were prasugrel, implying that American physicians preferred prasugrel over ticagrelor.

About $45 \%$ Chinese patients and $70 \%$ American patients received $\geq 80 \%$ of 365 DDDs' statin (table 3 ). American patients are treated with higher daily doses of statins and the continuity tends to be higher (multivariate-adjusted OR (AOR) (95\% confidence interval $(95 \%$ CI) ), 3.01 (2.23 to 4.06), $\mathrm{P}<0.001$, table 4), but their adherence (MPR $280 \%$ ) appears to be poorer (AOR (95\% CI) for American over Chinese, 0.37 (0.23 to 0.58), $\mathrm{p}<0.001$, in table 4$)$.

The sensitivity analyses, adjusted for PS weight for ATT and ATE, demonstrate the similar patterns (table 4). For antiplatelet agents, there are significant between-group differences $(p<0.001)$ regarding the continuity and adherence levels between Chinese and American patientsAmerican patients are more adherent to antiplatelet agents (table 4) and receive higher doses (table 3). In table 5, Chinese patients demonstrate a lower discontinuation rate for statins but a higher rate for antiplatelet agents.

\section{DISCUSSION}

Our study assessed the between-group difference in the medication use patterns of LLDs and antiplatelet agents between US Optum and HK CDARS databases. We found Chinese patients demonstrated a higher adherence level with statins than American patients, while discontinuation from stains was more frequent in Optum patients. Our study was not the first to identify this. According to a previous study among high cardiovascular risk patients in the USA, $53.0 \%$ of patients withdrew from statin therapy throughout 15 months' follow-up. ${ }^{38}$ The likelihood existed that the American patients in our cohort discontinued the statins due to the high incidence of safety events, however, the likelihood should be minor in this case given that Asian patients were more liable for the intolerance to statin. ${ }^{39}$

The continuity level, measured by $\geq 80 \%$ of 1 year DDDs, of HK patients was lower because the dosing scheme of statins in Chinese patients was mainly dominated by low-to-moderate potency statin. DDD could be taken as a proxy for average annual consumption per person and had the advantage of being internationally recognised as assigned and published by the $\mathrm{WHO}^{4041}$; however, it had the major limitations that across different ethnic or risk groups, the recommended dosing scheme could vary, and purely relying on an international standardised measure of dose equivalence for all patients can, therefore, be misleading. ${ }^{42}$ For example, the DDD by the WHO ATC/ DDD standard for simvastatin was $30 \mathrm{mg}$ per day, which belonged to moderate-potency statin. However, according to the most recent ACC/AHA guidelines ${ }^{43}$ ACS patients 


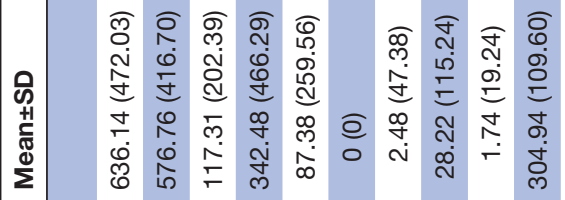

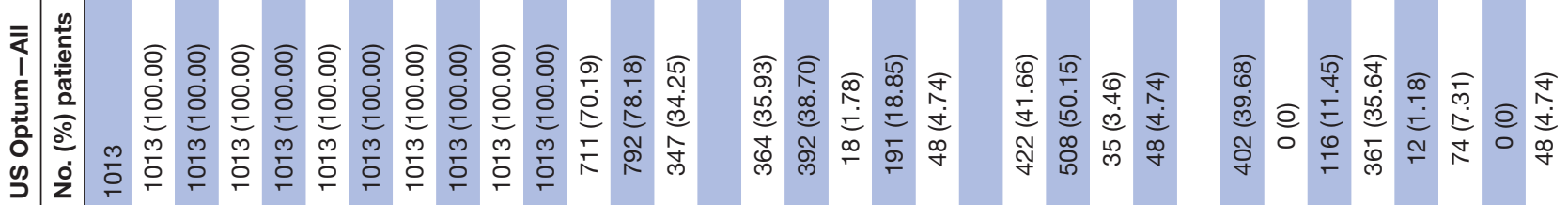

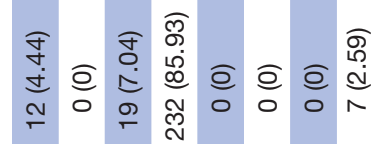

咅

兵

g<smiles>C1=CCCCC1</smiles>

के

苋

क 今े

올 
should be treated with the high-potency statins as long as they were $<75$ years old and could tolerate. As observed, $41.66 \%$ of US patients were put on high-potency statins after their index PCI, which would result in more DDDs than an annual use of 365 DDDs for each patient, therefore denoting a higher continuity while a lower adherence level was present.

Moreover, in Optum, atorvastatin was commonly used in treating American patients. For a long while, no statins could denote any advantage over atorvastatin (Lipitor). ${ }^{44}$ The other higher-potency statin rosuvastatin (Crestor) failed to bring in any incremental benefits and so was less likely to take the market share ${ }^{44}$ at this stage. It was obvious that by average American patients consumed more statin drugs than HK Chinese patients. Our observation that $\mathrm{HK}$ patients were treated with less intensive and lower quantity of statins was not surprising: the safety issue of high-potency statins among Chinese patients was widely discussed ${ }^{3945}$ and meanwhile Chinese patients were labelled as 'hyper-responders' to low-to-moderate potency statin. Compared with Caucasians, Chinese achieved a higher blood concentration of statins for a given dose of statin. ${ }^{46}$ The most remarkable study challenging the use of the high-potency statins in Chinese was HPS2-THRIVE trial, ${ }^{48}$ which found the rate of myopathy on simvastatin alone was higher among Chinese individuals, but they also concluded this 'small absolute excess' of myopathy with simvastatin $40 \mathrm{mg}$ daily was less likely to outweigh the cardiovascular benefits in the high-risk group. The solid evidence, in terms of results from clinical trials, to support the higher risk of severe statin toxicity in Chinese was lacking. However, the fact we could assure was, both in mainland China ${ }^{49}$ and $\mathrm{HK}^{50}$ the majority of the patients seldom received high-potency statins, and meanwhile substantial numbers of high-risk patients did not reach the lipid goals. Although the cardiologists in HK hospital setting mainly referred to the western guidelines, very interestingly, we found physicians were less willing to prescribe high-potency statins to local patients. Moreover, the use of non-statin LLDs was rare in HK patients. At the time of our study period, the routine use of non-statin lipid-lowering agents was not fully encouraged. However, the 2017 Focused Update ${ }^{51}$ of ACC guideline made it clear that addition of non-statin lipid-lowering agents to maximally tolerated statin therapy was recommended among patients with clinical ASCVD when the additional LDL lowering should be achieved. Therefore, we suggested the future studies look at the influence of new guidelines on the medication use pattern of non-statin LLDs.

The drug-specific adherence pattern was identified in HKlocal management. Compared with American patients, HK patients were less adherent to antiplatelet agents but better adherent to statins. Uncertainty remained among cardiologists regarding the uptake of 1 year antiplatelet agents after ACS. In HK hospitals, the recommended treatment duration of clopidogrel after PCI has gradually increased over these years from 1 to 6-12 months, which may explain why the adherence with antiplatelet 
Table 4 OR for good adherence and continuity with statins and antiplatelet agents

\begin{tabular}{|c|c|c|c|}
\hline & $\mathbf{O R}^{*}$ & $95 \% \mathrm{Cl}$ & $P$ value \\
\hline \multicolumn{4}{|l|}{ Good adherence (MPR $\geq 80 \%$ ) with statins } \\
\hline US Optum versus HK CDARS (adjusted†) & 0.37 & (0.23 to 0.58$)$ & $<0.001$ \\
\hline US Optum versus HK CDARS (PS weights for ATE $\ddagger$ ) & 0.42 & $(0.22$ to 0.81$)$ & 0.009 \\
\hline US Optum versus HK CDARS (PS weights for ATT§) & 0.44 & (0.21 to 0.92$)$ & 0.030 \\
\hline \multicolumn{4}{|l|}{ Good continuity (DDD/year $\geq 80 \% \times 365$ days) with statins } \\
\hline US Optum versus HK CDARS (adjusted†) & 3.01 & (2.23 to 4.06$)$ & $<0.001$ \\
\hline US Optum versus HK CDARS (PS weights for ATE $\ddagger$ ) & 4.28 & (2.94 to 6.22 ) & $<0.001$ \\
\hline US Optum versus HK CDARS (PS weights for ATT§) & 4.90 & (3.25 to 7.38$)$ & $<0.001$ \\
\hline \multicolumn{4}{|l|}{ Good adherence (MPR $\geq 80 \%$ ) with antiplatelet agents } \\
\hline US Optum versus HK CDARS (adjusted†) & 2.23 & (1.60 to 3.12$)$ & $<0.001$ \\
\hline US Optum versus HK CDARS (PS weights for ATE $\ddagger$ ) & 2.09 & (1.40 to 3.13$)$ & $<0.001$ \\
\hline US Optum versus HK CDARS (PS weights for ATT§) & 2.07 & (1.31 to 3.26$)$ & 0.002 \\
\hline \multicolumn{4}{|c|}{ Good continuity (DDD/year $\geq 80 \% \times 365$ days) with antiplatelet agents } \\
\hline US Optum versus HK CDARS (adjusted†) & 1.87 & (1.36 to 2.56$)$ & $<0.001$ \\
\hline US Optum versus HK CDARS (PS weights for ATE $\ddagger$ ) & 1.70 & (1.16 to 2.49$)$ & 0.007 \\
\hline US Optum versus HK CDARS (PS weights for ATT§) & 1.64 & (1.06 to 2.54$)$ & 0.026 \\
\hline
\end{tabular}

${ }^{*}$ Relative to Chinese patients in HK CDARS.

†Adjusted for sex, age, comorbidities of diabetes and hypertension and prior cardiovascular disease. ‡PS weights for ATE.

§PS weights for the ATT.

ATE, average treatment effect; ATT, average treatment effect for the treated; CDARS, Clinical Data Analysis and Reporting System;

$\mathrm{Cl}$, confidence interval; DDD, defined daily doses; MPR, medical possession ratio; OR, odds ratio; PS, propensity score.

agents appeared less in HK. It was less likely that difference in drug price was the significant contributor to this, as clopidogrel (HKD 0.91 (USD 0.12)/75 mg) in local pharmacy was available as inexpensive generics, whose price was comparable to that of moderate-potency simvastatin (HKD 0.245 (USD 0.031) / $40 \mathrm{mg}$ ). Previous qualitative research proposed several possibilities for patients' discontinuation behaviour ${ }^{52}{ }^{53}$ : The patients' compliance with statins appeared to link to the patients' belief about cholesterol level and LLDs, and inefficient physicianpatient communication. ${ }^{52} 53$ Another study ${ }^{54}$ found that the major reasons for LLD discontinuation were adverse side events, and the less common reasons were the drug price, and the mistrust, confusion and preference for other therapies of the patient. Patients who discontinued LLDs were more likely to challenge the necessity of LLDs than patients who discontinued clopidogrel. ${ }^{54}$ Reasons for discontinuing the clopidogrel included the confusion

\begin{tabular}{|c|c|c|c|}
\hline & $\mathbf{H R}^{*}$ & $95 \% \mathrm{Cl}$ & $P$ value \\
\hline \multicolumn{4}{|l|}{ Discontinuation with statins } \\
\hline US Optum versus HK CDARS (adjusted†) & 2.95 & (2.05 to 4.24$)$ & $<0.001$ \\
\hline US Optum versus HK CDARS (PS weights for ATE $\ddagger$ ) & 3.51 & (2.12 to 5.81$)$ & $<0.001$ \\
\hline US Optum versus HK CDARS (PS weights for ATT§) & 3.76 & (2.06 to 6.88$)$ & $<0.001$ \\
\hline \multicolumn{4}{|l|}{ Discontinuation with antiplatelet agents } \\
\hline US Optum versus HK CDARS (adjusted†) & 0.55 & (0.43 to 0.69$)$ & $<0.001$ \\
\hline US Optum versus HK CDARS (PS weights for ATE $\ddagger$ ) & 0.62 & (0.48 to 0.82$)$ & 0.001 \\
\hline US Optum versus HK CDARS (PS weights for ATT§) & 0.65 & (0.48 to 0.88$)$ & 0.006 \\
\hline
\end{tabular}

${ }^{*}$ Relative to Chinese patients in HK CDARS.

†Adjusted for sex, age, comorbidities of diabetes and hypertension and prior cardiovascular disease.

‡PS weights for ATE.

§PS weights for the ATT.

ATE, average treatment effect; ATT, average treatment effect for the treated; CDARS, Clinical Data Analysis and Reporting System; HR, hazard ratio; PS, propensity score. 
on the duration of the therapy, possible side effects, and drug price ${ }^{54}$ In the study, the possibility existed that statins were better prescribed than antiplatelet agents in HK due to the availability of lab results to monitor patients' lipid levels and slow uptake of antiplatelet agents in the local clinical practice.

Additionally, the choice of antiplatelet agents differed substantially between two cohorts. In HK, clopidogrel was the most dominant. In contrast, more American patients were prescribed prasugrel as the first antiplatelet agent but very few took ticagrelor. Ticagrelor was not available in the USA until July 2011, which could greatly affect its prescribing from the other two antiplatelet agents. The early guidelines in $2011,{ }^{55}$ during our study period, did not endorse any of antiplatelet agents over another, because at that time, the clinical benefits of prasugrel over ticagrelor was not that confirmative. From our prior research in $\mathrm{HK}^{56}{ }^{56}$ we found the ticagrelor was more cost-effective than clopidogrel, however, the uptake of ticagrelor was still slow, probably because the price of ticagrelor (11 HKD (USD 1.41) /90 mg per tablet) was much higher than clopidogrel (0.91 HKD(USD 0.12) $/ 75 \mathrm{mg}$ ).

There is no golden standard in justifying which country is doing better. Our research studied the patients in 2011-2012. There were several guideline updates to the management of ACS patients since this research was conducted, all of which could affect the prescribing practices of these medications. From the current research, we could hardly answer the question if HK physicians were 'policy-driven' and if they were following the cost-saving strategies when they prescribed. It was very likely the medication adherence and continuity in HK was determined by the system and the doctors rather than the patient when it was measured this way. The cost-saving prescription behaviour was widely reported ${ }^{10-12}$ to our local environment.

Some of the limitations bore mentioning. Due to the availability of data in Optum, we lacked a complete record of lab results and the exact reason for patients' discontinuation. Evaluation of adherence and continuity based on a patient's refill history could hardly account for the actual drug use and taking the medication in the correct way, instead, it was just an objective and relatively easy measurement. ${ }^{57}$ The reason for patients' actual medication use was beyond our reach. Some known predictors for the medication use, for example, socioeconomic status and ethnic minority status, were beyond our reach.

\section{CONCLUSION}

Our research shed some light on the real-world medication use pattern of HK-we found relatively good adherence with statins but bad adherence with antiplatelet agents in HK Chinese patients, compared with the privately insured patients in the USA. We identified the needs for the future qualitative and quantitative research to explore the factors leading to the medication use pattern.

\section{Author affiliations}

${ }^{1}$ Peninsula Clinical School, Monash University, Clayton, Victoria, Australia ${ }^{2}$ University of Southern California Sol Price School of Public Policy, Los Angeles, California, USA

${ }^{3}$ Department of Medicine \& Therapeutics Faculty of Medicine, The Chinese University of Hong Kong, Hong Kong, China

${ }^{4}$ Prince of Wales Hospital, Hospital Authority, Hong Kong, China

${ }^{5}$ School of Pharmacy, The Chinese University of Hong Kong, Hong Kong, China

Acknowledgements This study used deidentified electronic health records data and claims data from the two databases Clinformatics Data Mart database (Optumlnsight, Eden Prairie, Minnesota, USA) and Hospital Authority (HA) Clinical Data Analysis and Reporting System (CDARS), respectively.

Contributors YW and VWYL conceived and designed this study. YW and JW collected the data. YW analysed the data and drafted the paper. MBN, BPY, JW, BT and VWYL revised the paper. VWYL and MBN led and supervised this study. All authors read and approved the final paper and contributed substantially to the project.

Funding The authors have not declared a specific grant for this research from any funding agency in the public, commercial or not-for-profit sectors.

Competing interests None declared.

Patient consent for publication Not required.

Ethics approval The study was approved by the Joint Clinical Research Ethics Committee of The Chinese University of Hong Kong and New Territories East Cluster (CUHK-NTEC), and the protocol was compliant to the Declaration of Helsinki.

Provenance and peer review Not commissioned; externally peer reviewed.

Data sharing statement Due to ethical concerns, supporting data cannot be made openly available.

Open access This is an open access article distributed in accordance with the Creative Commons Attribution Non Commercial (CC BY-NC 4.0) license, which permits others to distribute, remix, adapt, build upon this work non-commercially, and license their derivative works on different terms, provided the original work is properly cited, appropriate credit is given, any changes made indicated, and the use is non-commercial. See: http://creativecommons.org/licenses/by-nc/4.0/.

\section{REFERENCES}

1. Kong X, Yang Y, Gao J, et al. Overview of the health care system in Hong Kong and its referential significance to mainland China. J Chin Med Assoc 2015;78:569-73.

2. Health Do. Health facts of Hong Kong 2017 Edition. 2017.

3. Statistics. NCfH. Health, United States, 2016: with chartbook on long-term trends in health. Hyattsville, MD, 2017.

4. Hospital Authority HK. Fees and Charges. 2018. http://www.ha.org. hk/visitor/ha_visitor_index.asp?Content_ID=10045\&Lang=ENG

5. Sen A. Universal healthcare: the affordable dream. The Guardian 2015;6:2015.

6. Wiley Online Library. A universal healthcare system: is it right for the United States? Nursing Forum. 2007.

7. Hay JW. Health care in Hong Kong: an economic policy assessment: Chinese University Press. 1992.

8. Wang EC, Choe MC, Meara JG, et al. Inequality of access to surgical specialty health care: why children with government-funded insurance have less access than those with private insurance in Southern California. Pediatrics 2004;114:e584-90.

9. Ayanian JZ, Kohler BA, Abe T, et al. The relation between health insurance coverage and clinical outcomes among women with breast cancer. N Engl J Med 1993;329:326-31.

10. Wong MC, Jiang JY, Lam AT, et al. Patterns of antihypertensive prescribing, discontinuation and switching among a Hong Kong Chinese population from over one million prescriptions. J Hum Hypertens 2008;22:714-6.

11. Leung R, Wong G, Lau J, et al. Prevalence of asthma and allergy in Hong Kong schoolchildren: an ISAAC study. Eur Respir J 1997;10:354-60.

12. Cheng SW, Ting AC, Tsang SH. Epidemiology and outcome of aortic aneurysms in Hong Kong. World J Surg 2003;27:241-5.

13. Finance and NHS/Medicines PalG. The Pharmaceutical Price Regulation Scheme 2014. 2013.

14. Ford ES, Capewell S. Coronary heart disease mortality among young adults in the U.S. from 1980 through 2002. J Am Coll Cardiol 2007;50:2128-32. 
15. Schoenenberger AW, Radovanovic D, Stauffer JC, et al. Acute coronary syndromes in young patients: presentation, treatment and outcome. Int J Cardiol 2011;148:300-4

16. Wong IO, Chan WS, Choi S, et al. Moral hazard or realised access to care? Empirical observations in Hong Kong. Health Policy 2006;75:251-61.

17. Hui KC, Tang SC, Yan BP, et al. Relationship Between Adherence To Post Percutaneous Coronary Intervention (Pci) Guidelines And Clinical Outcomes Of Post-Pci Patients In Hong Kong - a 5-year retrospective cohort study. Value Health 2015;18:A404.

18. Blais JE, Chan EW, Law SWY, et al. Trends in statin prescription prevalence, initiation, and dosing: Hong Kong, 2004-2015. Atherosclerosis 2019;280:174-82.

19. Luk HH, Pang J, Li LS, et al. Use of antiplatelet drugs in the stroke unit of a Hong Kong hospital. Pharm World Sci 2005;27:258-62.

20. Ray KK, Kastelein JJ, Boekholdt SM, et al. The ACC/AHA 2013 guideline on the treatment of blood cholesterol to reduce atherosclerotic cardiovascular disease risk in adults: the good the bad and the uncertain: a comparison with ESC/EAS guidelines for the management of dyslipidaemias 2011. Eur Heart J 2014;35:960-8.

21. Poon RT, Cheung TT, Kwok PC, et al. Hong Kong consensus recommendations on the management of hepatocellular carcinoma. Liver Cancer 2015;4:51-69.

22. Joint committee for guideline revision. 2016 Chinese guidelines for the management of dyslipidemia in adults. $J$ Geriatr Cardiol 2018:15:1-29.

23. Drozda JP, Ferguson TB, Jneid H, et al. 2015 ACC/AHA Focused update of secondary prevention lipid performance measures: a report of the American College of Cardiology/American Heart Association Task Force on Performance Measures. J Am Coll Cardiol 2016:67:558-87.

24. Kesselheim AS, Huybrechts KF, Choudhry NK, et al. Prescription drug insurance coverage and patient health outcomes: a systematic review. Am J Public Health 2015;105:e17-e30.

25. Desai $\mathrm{V}, \mathrm{Nau} \mathrm{D}$, Conklin M, et al. Impact of environmental factors on differences in quality of medication use: an insight for the medicare star rating system. J Manag Care Spec Pharm 2016;22:779-86.

26. Government HK. LCQ13: Resource allocation of Hospital Authority. 2017.

27. Lee HY, Cooke CE, Robertson TA. Use of secondary prevention drug therapy in patients with acute coronary syndrome after hospital discharge. J Manag Care Pharm 2008;14:271-80.

28. Varas-Lorenzo C, Castellsague J, Stang MR, et al. Positive predictive value of ICD-9 codes 410 and 411 in the identification of cases of acute coronary syndromes in the Saskatchewan Hospital automated database. Pharmacoepidemiol Drug Saf 2008;17:842-52.

29. Lenihan CR, Montez-Rath ME, Winkelmayer WC, et al. Multivessel coronary revascularization and outcomes in kidney transplant recipients. Transpl Int 2013;26:1080-7.

30. Wettermark B, Elseviers M, Almarsdóttir AB, et al. Introduction to drug utilization research. Drug Utilization Research: Methods and Applications 2016:1-12.

31. Larsen J, Vaccheri A, Andersen M, et al. Lack of adherence to lipid-lowering drug treatment. A comparison of utilization patterns in defined populations in Funen, Denmark and Bologna, Italy. Br J Clin Pharmacol 2000;49:463-71.

32. Reach G. Treatment adherence in patients with gout. Joint Bone Spine 2011;78:456-9.

33. Byfield SA, McPheeters JT, Burton TM, et al. Persistence and compliance among U.S. patients receiving pazopanib or sunitinib as first-line therapy for advanced renal cell carcinoma: a retrospective claims analysis. J Manag Care Spec Pharm 2015;21:515-22.

34. Sinnott SJ, Polinski JM, Byrne S, et al. Measuring drug exposure: concordance between defined daily dose and days' supply depended on drug class. J Clin Epidemiol 2016;69:107-13.

35. Guo S, Fraser MW. Propensity score analysis: Statistical methods and applications: Sage Publications. 2014.

36. Grilli L, Rampichini C. Propensity scores for the estimation of average treatment effects in observational studies. Training Sessions on Causal Inference, Bristol. 2011:28-9.

37. Austin PC. An introduction to propensity score methods for reducing the effects of confounding in observational studies. Multivariate Behav Res 2011;46:399-424.
38. Lin I, Sung J, Sanchez RJ, et al. Patterns of statin use in a real-world population of patients at high cardiovascular risk. J Manag Care Spec Pharm 2016;22:685-98.

39. Liao JK. Safety and efficacy of statins in Asians. Am J Cardiol 2007;99:410-4.

40. Malo S, Aguilar-Palacio I, Feja C, et al. Different approaches to the assessment of adherence and persistence with cardiovascular-disease preventive medications. Curr Med Res Opin 2017;33:1329-36.

41. Wertheimer Al. The defined daily dose system (DDD) for drug utilization review. Hosp Pharm 1986;21:233-4.

42. Rønning M, Blix HS, Harbø BT, et al. Different versions of the anatomical therapeutic chemical classification system and the defined daily dose--are drug utilisation data comparable? Eur J Clin Pharmacol 2000;56(9-10):723-7.

43. Stone NJ, Robinson J, Lichtenstein AH, et al. ACC/AHA guideline on the treatment of blood cholesterol to reduce atherosclerotic cardiovascular risk in adults. Circulation 2013.

44. Jackevicius CA, Chou MM, Ross JS, et al. Generic atorvastatin and health care costs. N Engl J Med 2012;366:201-4.

45. Li DQ, Kim RB, McArthur E, et al. Statin Safety in Chinese: a population-based study of older adults. PLOS One 2016;11:e0150990.

46. Wang P. Statin dose in Asians: is pharmacogenetics relevant? Pharmacogenomics 2011;12:1605-15.

47. Tomlinson B, Chan P, Liu ZM. Statin Responses in Chinese Patients. $J$ Atheroscler Thromb 2018;25:199-202.

48. Haynes R, Jiang L, Hopewell JC, et al. HPS2-THRIVE randomized placebo-controlled trial in 25673 high-risk patients of ER niacin/laropiprant: trial design, pre-specified muscle and liver outcomes, and reasons for stopping study treatment. Eur Heart $J$ 2013;34:1279-91.

49. Wang F, Ye P, Hu D, et al. Lipid-lowering therapy and lipid goal attainment in patients with metabolic syndrome in China: subgroup analysis of the dyslipidemia international study-China (DYSIS-China). Atherosclerosis 2014;237:99-105.

50. Wang Y, Yan BP, Nichol MB, et al. Real-world study of low-density lipoprotein cholesterol levels and cardiovascular outcomes in Chinese: A retrospective cohort study in post-percutaneous coronary intervention acute coronary syndrome patients. Int $\mathrm{J}$ Cardiol 2017;249:18-24.

51. Lloyd-Jones DM, Morris PB, Ballantyne CM, et al. 2017 Focused Update of the 2016 ACC Expert consensus decision pathway on the role of non-statin therapies for Idl-cholesterol lowering in the management of atherosclerotic cardiovascular disease risk: a report of the American College of cardiology task force on expert consensus decision pathways. J Am Coll Cardiol 2017;70:1785-822.

52. Tolmie EP, Lindsay GM, Kerr SM, et al. Patients' perspectives on statin therapy for treatment of hypercholesterolaemia: a qualitative study. Eur J Cardiovasc Nurs 2003;2:141-9.

53. Todd A, Holmes H, Pearson S, et al. 'I don't think I'd be frightened if the statins went': a phenomenological qualitative study exploring medicines use in palliative care patients, carers and healthcare professionals. BMC Palliat Care 2016;15:13.

54. Garavalia L, Garavalia B, Spertus JA, et al. Exploring patients' reasons for discontinuance of heart medications. J Cardiovasc Nurs 2009;24:371-9.

55. Wright RS, Anderson JL, Adams CD, et al. 2011 ACCF/AHA focused update of the guidelines for the management of patients with unstable Angina/Non-ST-Elevation myocardial infarction (updating the 2007 guideline): a report of the American College of Cardiology Foundation/American Heart Association task force on practice guidelines developed in collaboration with the American College of Emergency Physicians, society for cardiovascular angiography and interventions, and society of thoracic surgeons. J Am Coll Cardiol 2011;57:1920-59.

56. Wang Y, Yan B, Liew D, et al. Cost-effectiveness of cytochrome P450 2C19* 2 genotype-guided selection of clopidogrel or ticagrelor in Chinese patients with acute coronary syndrome. The pharmacogenomics journal 2017.

57. Krumme AA, Franklin JM, Isaman DL, et al. Predicting 1-year statin adherence among prevalent users: a retrospective cohort study. $J$ Manag Care Spec Pharm 2017;23:494-502. 